\title{
Introduction to Electronic Properties and Dynamics of Organic Complexes as Self-Assembled Monolayers
}

\author{
Maddalena Pedio and Barbara Ressel \\ Additional information is available at the end of the chapter
}

http://dx.doi.org/10.5772/68111

\begin{abstract}
Self-assembled monolayers (SAMs) of organic-conjugated transition metal complexes on surfaces is a focus of both device engineering and basic science, since it is a key factor in nearly all important aspects of device performances, including operation voltages, degradation, and efficiency. The huge amount of literature results related to the first monolayer, and reorganization and self-assembling processes are due to the general accepted result that structural and chemical properties of the first monolayer are the key parameters for controlled thin film growth. Optical and magneto-electronic properties are intimately connected, and the accurate determination of electronic levels, excitation, and relaxation dynamics is mandatory for the optimization of electronic, photovoltaic, and opto-electronic devices. Quite a number of electronic states is generated by the interaction of light with complex organic molecules. Time-resolved spectroscopies are a new investigation tool that gives the possibility of correctly addressing their origin and life time. Examples of prototypical systems are presented and discussed. We review on complementary techniques, trying to single out how different approaches are fundamental to fully characterize these complex systems.
\end{abstract}

Keywords: self-assembled monolayer (SAM), surface structures molecular layers, nanotechnology, electronic properties, spectroscopies, time resolved

\section{Introduction}

The fundamental understanding of the molecular solids properties and the controlled ordering processes of organic complexes received a paramount interest for their promising applications. The current challenge in nanostructured materials consists of the understanding of ever smaller scale and in exploiting their functionality at the relevant length, time, and energy scales. 
In organic semiconductors, their molecular aggregation and crystal packing in solid state induce unique properties that differ from those of the single molecule components or their liquid solutions, affecting their structural, optical, and electronic-magnetic properties. The understanding and the control of the processes determining the dynamic properties of organic semiconductors are crucial for further development of organic electronics and other technological applications.

The level of effort placed on the study of transition metal complexes (TMCs) [1] and their single layers on surfaces has grown steadily in the last few decades. Large molecules deposition on surfaces leads to a high variety of interactions. The bond strength spreads from very weak VdW to no covalent or strong chemical bonding. Normally, organic-inorganic are multiphase systems with complex phase diagrams. The experience in these decades has stated that molecule-substrate must be viewed as a whole. Even in weak-bounded molecular layers [2], the simple change of symmetry induced by adsorption and the induced dipoles can lead to modifications of the molecular and metallic states, affecting the electronic structure at the interface [3-6]. The static and dynamic characterization on different time scales are crucial for the comprehension of such complex systems, as well as the understanding of the mutual correlation among the structural, opto-magnetic, and electronic properties. In view of applications, thin and ultrathin films of TMCs are of great current interest, and the control of the thin films' growth and crystallinity is of crucial importance to optimize the performance of future electronic devices. To acquire the ability to design materials with selected properties requires a deep understanding of the proper molecular precursors characteristics used as building blocks and of the modifications induced when deposited in films.

In this chapter, case systems involving TMCs will be addressed, focusing the discussion on their dynamical properties.

Among TMCs, the tetrapyrrole complexes, such as metallo-porphyrins, phthalocyanines, and dipyrrins, are ubiquitous molecules, and their presence spans the geological world with etioporphyrins and up to biology with hemoproteins. They serve as agents to transport molecules (hemoproteins) or electrons (chlorophylls) and are also important as photochemical agents with chlorophyll antennae. Synthetic porphyrins and phthalocyanines are used in the phototherapy of cancer or in solar cells, through virtue of their unique photophysical properties, and are versatile building blocks for the realization of molecular materials, which exhibit various light-driven chemical reactions. A multitechnique approach is necessary to extract and enlighten the interrelation between structural details and the opto-electronic properties [7]. A number of reviews [3-6, 8, 9] and accounts dealing with specific aspects of organic layers on different classes of substrates, conductive or semiconductors are present in the literature.

Among the organic semiconductors, those formed by tetrapyrrole metal complexes (such as porphyrins and phthalocyanines) are found in a wide variety of technological applications including electronics, biological, and chemical contexts. Porphyrins often act as metal binders and find applications in dye-sensitized solar cells, in molecular magnets, oxygen sensors, etc. To fabricate organic photovoltaic (OPV) solar cells [10] with high conversion efficiency, it has been proved that it is crucial to control the crystal structure, crystallinity, and molecular 
orientation of the thin films used, because the diffusion length of excitons and/or charge carriers, electrons, and holes in their crystalline films becomes longer than in their amorphous form. These factors became even more crucial in the single layer interacting with substrates, as discussed in the next paragraph.

We review the recent findings on the excitation and relaxation dynamics of TM tetrapyrrole single layer (metal octaethyl porphyrins, MOEP; metal tetraphenyl porphyrins, MTPP) and phthalocyanines (MPcs) by means of time-resolved optical and photoelectron spectroscopies, enlightening the aspects of molecular reorganization chemistry on model solid surfaces, with a focus on molecule-substrate bond and energy level alignment.

\section{From single molecules to aggregates}

The knowledge of the molecular properties in the gas phase is mandatory for the understanding of the whole set of molecular applications. The spectroscopic characterization of molecular energy levels in vapor has had enormous improvements in the last decades [11]. In TMC, the central metal ion and its nd orbital occupancy strongly affect the complex properties. For example, in transition metal phthalocyanine (MPc) [12], the comparison between the series of TMCs versus $3 \mathrm{~d}$ occupancy shows that in choosing the proper metal, the filled orbitals and in particular the highest occupied molecular orbital (HOMO) allow to tune their binding energy to engineer their transport and optical gaps.

In organic semiconductors, the solid aggregation induces unique optical and electronic-magnetic properties, which often differ from those of the single molecule components or their liquid solutions, affecting their structural, optical, and electronic-magnetic properties as discussed in the following sections. The reason is qualitatively illustrated in Figure 1, where the energies of the so-called frontier orbitals, highest occupied molecular orbital HOMO and the lowest unoccupied molecular orbital (LUMO) result perturbed in solids due to intermolecular interactions.

Strong theoretical efforts have been applied in the last decades to characterize the geometry, electronic, and optical properties of the TMC, accompanied by a robust experimental set of
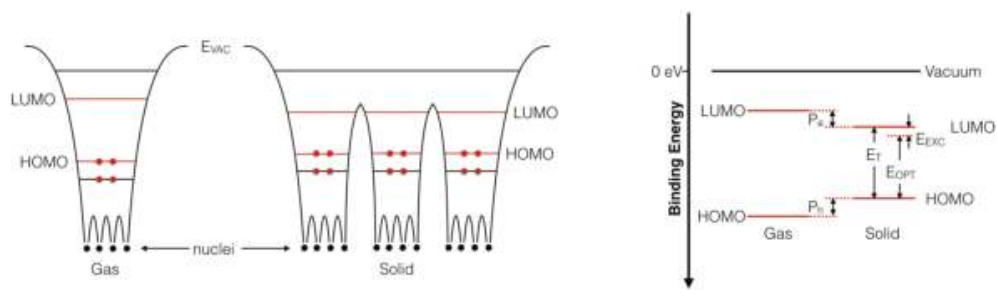

Figure 1. Left: Energy levels in the potential wells of an isolated molecule and molecular solids. Right: The corresponding energy level scheme shows the frontier orbital HOMO-LUMO gap in gas and the optical $\left(\mathrm{E}_{\mathrm{OPT}}\right)$ and transport $\left(\mathrm{E}_{\mathrm{T}}\right)$ gaps in solid, while $\mathrm{E}_{\mathrm{EXC}}$ is the exciton binding energy [9]. 
data on free molecules, solutions, and solid aggregates [4, 13, 14]. The aggregation enhances the intermolecular $\pi-\pi$ stacking, facilitating the intramolecular charge transport. The understanding and the control of the processes determining the magneto-electronic and dynamic properties of organic semiconductors are crucial to the further development of organic electronics and other technological applications. Better crystal quality with large domains increases properties like conductivity by more than one order of magnitude. It has been proved how critical it is to control the crystal structure, crystallinity, and molecular orientation of the thin films because the diffusion length of excitons and/or charge carriers, electrons, and holes in their crystalline films becomes longer than in their amorphous form. Intermolecular interactions, dishomogeneities, and grains within the molecular solids are related to the $\pi-\pi$ intermolecular interactions exhibiting strong electron-phonon coupling, important for the charge and exciton transport in organic devices [15].

Molecular crystalline materials can be altered by manipulating spin, electron transfer, proton transfer, molecular structure, and orientation, leading to dynamical switchable properties [16] by external stimuli such as light, electric field, and temperature. The precise control of the switching properties is the focus of recent frontier developments. For single layer, the dynamics study is still to be systematically understood due to the experimental difficulties. Recent results will be discussed in Section 4 .

\subsection{Transition metal complexes}

Transition metal coordination complexes are conjugated molecules that consist of a central metal atom or ion (coordination center) and a surrounding array of bound molecules or ions, ligands, or complex agents. Common coordination geometries are shown in Figure 2. Properties of TMCs include absorption and transmission of visible light (highly colored), multiple oxidation states of the metals, paramagnetism dependent on the nd electronic configuration of the metal ion, metal oxidation state and on ligand field, reactivity.

Here, we mainly focus on the large planar macrocycle complexes, tetrapyrrole molecules metallo porphyrins (MOEP, MTPP), and phthalocyanines (MPcs), whose specific functionalities are related to the presence in the macrocycle of the metal center in Figure 2. Porphyrin derivative complexes are ubiquitous molecules in nature and present an enormous variety of properties $[10,17]$. This pervasiveness arises from their special tetrapyrrole planar structure

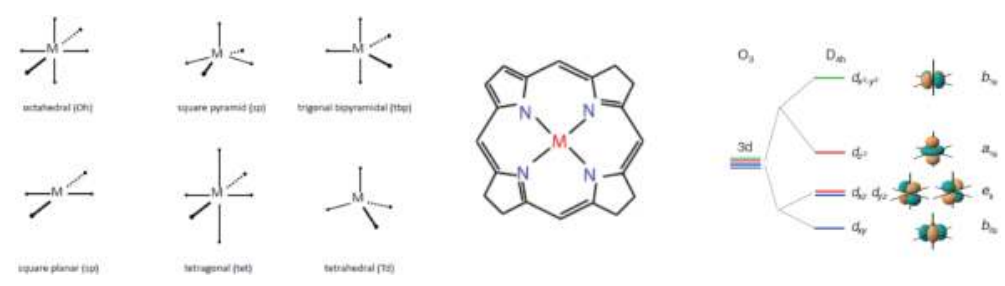

Figure 2. Left: Common coordination geometries in TM complexes and the structure of the porphyrin macrocycle common to the tetrapyrrole complexes. Right: The $3 \mathrm{~d}$ orbital splitting induced by a D4h square planar crystal field, adapted with permission from Ref. [18], copyright 2012 American Physical Society. 
with a metal atom bound to four $\mathrm{N}$ atoms in the macrocycle and from its stable $\pi$-conjugated system. The organic ligand of metallo-organic molecules affects the electronic states of the metallic ion. Central metallic ions atomic orbitals are hybridized with the organic ligand in the macrocycle, leading to molecular orbitals with a partial metallic character. The $3 \mathrm{~d}$ orbitals of the transition metal ion get split by the molecular tetragonal D4h symmetry crystal field, giving rise to the $3 d$ character molecular orbitals indicated in Figure 2 see for example Refs. [18, 19].

The large aromatic nature of tetrapyrroles provides the basis for their unique absorption properties, for example, the typical UV/Vis spectra of porphyrins exhibit absorptions in two regions termed the Soret or B band ( 380-420 nm), corresponding to transition from ground state to the second excited state, and the $Q$ bands $(\sim 500-800 \mathrm{~nm})$ related to the first excited state (Figure 3). This is a result of the splitting of the main frontier molecular orbitals, the highest occupied molecular orbital (HOMO) and the lowest unoccupied molecular orbital (LUMO). The electronic structure of porphyrins can be described by the semi-quantitative Gouterman's four-orbital model that considers the two HOMOs (labelled as a1 $u$ and $\mathrm{a} 2 u$ ) and LUMOs (labelled $e g$ ) of the porphyrin (Hückel theory) and mixes the four possible optical excitations between them using configuration interaction theory to account for electron interaction [17].

The surrounding groups present around the porphyrin ring have a detectable effect on the binding energy and shape of the molecular orbitals and the $3 d$ electronic configuration through conjugation. This is reflected in spectroscopic data as discussed, for instance, in the case of Ni complexes [21], where core level photoemission and X-ray absorption spectra of $\mathrm{Ni}, \mathrm{C}$, and $\mathrm{N}$ indicate that different charge transferred occurs in the different tetrapyrrole complexes, at the metal-to-ligand bonds, from the occupied Ni $3 d_{x z^{\prime}} d_{y z}\left(\mathrm{e}_{\mathrm{g}}\right)$ orbitals to the unoccupied N $2 \pi \pi^{*}\left(\mathrm{e}_{\mathrm{g}}\right)$ orbitals, hence inducing changes in the back donation among the different molecules, from NiOEP to NiPc. Other examples are present in the literature, for example, for Fe complexes [22].
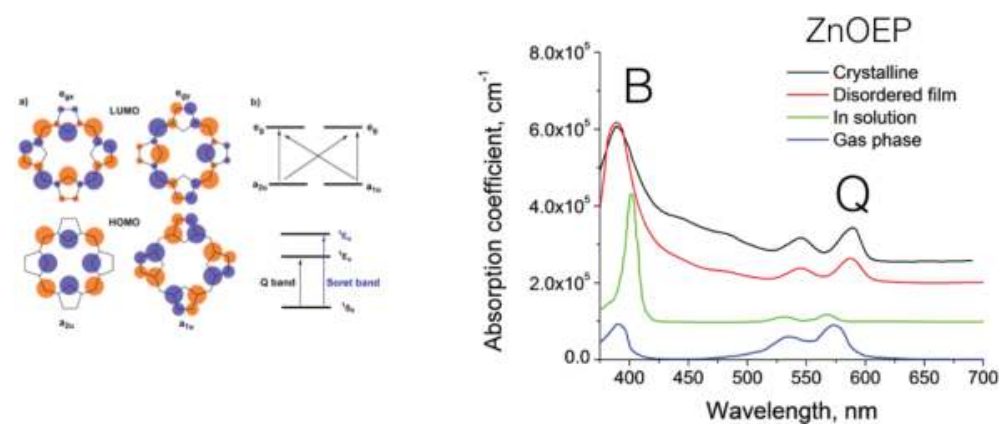

Figure 3. Simplified representation of the one electron transitions from HOMO to LUMO, related to their absorption spectra B (Soret) and Q band regions (from ref [17]) in the four orbital-model. Example of crystalline and disordered optical absorption for $\mathrm{ZnOEP}$ compare with the $\mathrm{ZnOEP}$ in toluene solution and gas phase data [20]. 
Tetrapyrrole derivatives aggregate [23] into a plethora of nanostructures such as films, crystals, tubes, rods, wires, nanoparticle spheres, and complex fractal-like or chiral patterns. By varying the choice of the metal center, the bonding and characteristics of the metalloporphyrin may be dramatically affected. Systematic experimental and theoretical evaluation of the porphyrin and MPc enlighten the role of the $3 d$ metal electronic states and occupancy in the conjugation of these complexes [18, 19, 24-27].

\subsection{Organic thin films}

The structural and electro-optical properties present a strong mutual influence and fine details' variation that can lead to strong alteration of macroscopic properties in the aggregates [28] as the transport and optical gap or the magnetic moments in the paramagnetic complexes. Moreover, organic thin films present structural polymorphism [29], depending on the molecular structure and the strength of the intermolecular interaction. Several works demonstrate the effect of different stacking structures at macroscopic scales [19, 30]. Octaethyl porphyrins (OEP) for example present a simple structure with the eight external ethyl groups in alternate orientation. Even in this relatively simple structure, the ruffling of the ethyl groups in aggregation or self-organization induces perturbation on the electronic properties as shown theoretically [31] for NiOEP. Moreover, the ordering is a crucial factor for applications: differences in optical absorption have been measured when comparing, for example, ZnOEP films with different degrees of long range order [32].

\subsection{Self-organization in the first layer}

The interest in the first monolayer of (organic $\pi$-conjugated systems or fullerene) molecules on single crystals adsorption is due to the general accepted result that structural and chemical properties of the first monolayer are keys for controlled film growth. Adsorption of complex molecules on metal and semiconductor interfaces may lead to the formation of highly ordered nanostructuration that can then be exploited for the fabrication of surface-supported nanostructures as molecular building blocks confined to 2D systems. Self-assembled monolayers are ordered molecular assemblies formed by the adsorption onto an inorganic or organic (heterojunctions) substrate. Self-assembly [33], in a general sense, might be defined as the spontaneous formation of complex hierarchical structures from pre-designed building blocks, typically involving multiple energy scales and multiple degrees of freedom. The knowledge of the interaction details of the first organic layer is mandatory: the investigation on the perturbation induced by the molecule-substrate interaction and how the isolated molecule properties are perturbed by intermolecular interactions in the assembling. Absorption and electron spectroscopies, together with scanning tunneling microscopy (STM), are precious tools that provide a clear framework of these complex systems.

In these studies, the question to be answered concerns the types of structures and phases that are formed and which parameters characterize the order:

- the self-assembling driving forces (what determines the growth kinetics and the growth regimes), 
- what are the internal (e.g., molecular conformers variation or substrate orientation) and the external (e.g., temperature) control parameters?

- the nature of the phase transitions and how do the various degrees of freedom and the different constituents of the molecule (headgroup, chain or backbone, endgroup) have an impact on the growth and the structure.

\section{Mutual effects of self-assembling on substrates and molecular conformers in tetrapyrrole complexes}

Organic films can be deposited by a variety of methods, as spin coating from solutions or ultrahigh vacuum (UHV) deposition. Here, we refer mainly to the latter method. Porphyrins and phthalocyanines, due to their very low room temperature and vapor pressure $\left(10^{-14}\right.$ Torr), can be sublimated in UHV and thus prepared and characterized as controlled grown molecular layers.

In the self-assembled monolayer (SAM), on ordered substrates, the break of translational symmetry at the interface induces a variation of the boundary conditions with relevant variation of the charge densities both in the molecule and in the substrate. The wave functions of these interface states have a large amplitude at the interface and decay quickly into both materials [3]. The mechanism of molecule surface interaction can be quite complex due to the balance between intermolecular binding forces and molecule-substrate interactions, involving a large number of sites due to the molecular size with respect to the unit cell of the substrates. This can result in either displacive substrate reconstructions and/or in complex structural deformation of the adsorbed molecules. The molecule-substrate system must be taken into account as a whole, and it is not possible to simply transfer molecular functionalities. The simulation of such systems is still a challenging task and deserves state-of-the art theoretical framework.

The tendency to form ordered self-organized structures on a variety of substrates and the existence of extended $\pi$-electron systems makes the study of tetrapyrrole TM complexes important from both fundamental and technological viewpoints.

In his recent detailed review, Gottfried [13] stressed that by means of the conjugated organic molecules, adsorption on metal substrates is possible to engineer the modification of interfacial properties, especially for planar tetrapyrrole complexes. The understanding of the interface electronic structure is therefore a prerequisite for insightful materials design.

\subsection{Ordering in the first layer and changes in molecules}

The self-assembly of TM complexes is driven by intermolecular interactions, which can be attractive or repulsive, depending on the molecular structure and the character of the surface chemical bond $([4,5,13]$ and references therein). An interface dipole takes place even in weakly interactive SAM interfaces (see also Figure 5), while chemical bonds facilitate the 
electron transfer between substrate and adsorbate. The resulting dipoles along the surface normal are repulsive and can over-compensate the lateral Van der Waals attraction. The preferred orientation is with the macrocycle parallel to the surface on conductive substrates. The lateral interactions are closely coupled to the vertical adsorbate-substrate distances, though their leading determination implies important experimental efforts. Other important aspects include the degree of mismatch between the molecular and the substrate symmetries, which can lead to adsorption-induced symmetry reductions in the molecule, and substrate-induced template effects, which affect the supramolecular arrangement. Moreover, in case of peripheral non-planar groups, the adsorption leads to a distortion of the molecular conformers as in MTPP, either because of their inherent non-planarity or because of intramolecular sterical hindrance. In the adsorbed state, this leads to a competition between the energy-decreasing maximization of the adsorbate-substrate contact, which forces the molecule to approach a planar conformation, and the energy-increasing intramolecular strain resulting from the distortion toward planarity.

As in isolated molecules, the central atom's electronic configuration and $3 d$ occupancy play a major role in the structural rearrangements of selected SAM [34] and in molecule-substrate bonds (chemisorbed/physisorbed) as found in MPc and porphyrins (review Table 4 Ref. [13]). Case systems are discussed in Section 3.3.

\subsection{Electronic structure and density of state at SAM interfaces}

The characterization of SAM by absorption and electron spectroscopies, together with scanning microscopies, provides unique information on the reorganization of the density of electron states (DOSs) at interfaces $([35,36]$ and refs therein). At interface, several processes take place in the delicate intermolecular, molecule-substrate interaction balance [37]: correlations between strength of molecule/metal interaction, average bonding distances, adsorption-induced molecular conformation changes leading to intramolecular dipoles, organic/metal interface dipoles, and the resulting unexpected energy level alignment. Interface energy level alignment, band bending, chemical reaction, charge transfer (CT), metal-organic interface dipole formation, internal molecular dipole, and Debye screening are all necessary factors to describe the single layer on surfaces. The reader is addressed to the numerous excellent papers and reviews present in the literature to get more details, for example, Refs. [4-6].

For the aim of this review, we just need to know that PES is a surface-sensitive technique that provides a direct insight of occupied density of states of electronic energy levels. It relies on the photoelectric effect: when a photon, whose energy $h v$ is larger than the sample work function (WF or ionization potential in case of gases), is shone on a sample, an electron is ejected with kinetic energy $\mathrm{KE}=h v-\mathrm{EB}-\mathrm{WF}$, where $\mathrm{EB}$ is the electron's binding energy. The combination of photoemission with inverse photoemission allows one to measure the electron affinity (EA), the work function $(\mathrm{WF})$, the transport gap, $\left(\mathrm{E}_{\mathrm{T}}\right)$, and the interface dipole $(\Delta)$, as indicated in Figure 4 [6]. In organic semiconductors, the ground state is characterized by the presence of electrons in the highest occupied molecular orbital (HOMO) and vacancies in the lowest unoccupied molecular orbital (LUMO). The absorption of a visible photon creates an 


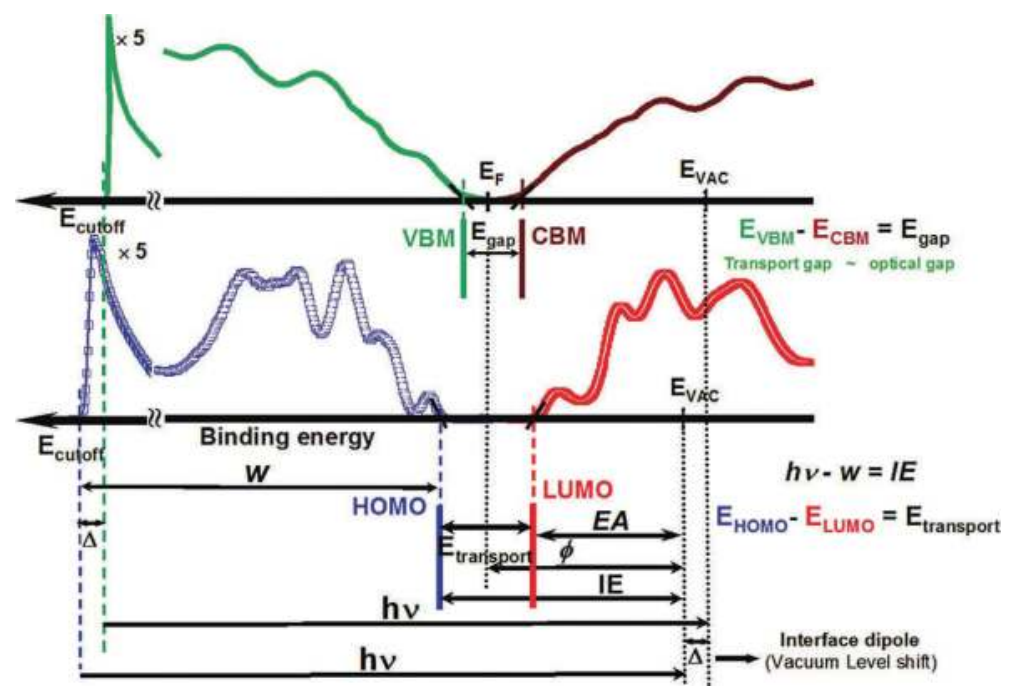

Figure 4. Combined photoemission and inverse photoemission spectra of an inorganic solid (upper panel) and thin organic film (lower panel) used to define the relevant quantities. Reprinted with permission from Ref. [6]. Copyright 2007-2017, American Chemical Society.

excited state, where an electron is promoted in the LUMO and a vacancy is left in the HOMO. However, this state is not stable because its energy is higher than that of the ground state and therefore it will decay.

Among the techniques that give access to magnetic moments, the $\mathrm{X}$-ray magnetic dichroic spectroscopy measurements with elemental sensitivity is widely used in the case of paramagnetic SAM $[18,19,38]$.

Moreover, imaging scanning microscopies such as scanning tunneling microscopy (STM) and scanning tunneling spectroscopy (STS) are giving a huge contribution to reveal the details of organic-inorganic systems. Among the excellent reviews present in the literature, we cite Ref. [39] dedicated to TMC interfaces.

Finally, a specific field of investigation is reserved to interface build by paramagnetic complexes, the spinterfaces, that deserves experimental methods sensible to the magnetic properties of the system. The study of the interconnections between the electronic configuration of the central atoms in the adsorbed TMC, the details of the interaction, and the resulting magnetic properties has recently produced new important results, useful in magneto-optical devices.

\subsection{Case systems and trends}

The tetraphenylporphyrin (TPP) complexes deposited on metal surfaces are one of the most studied self-organized systems in the literature. They present the tendency to polymorphism 
related to the external phenyl orientations and the consequent perturbation of the macrocycle planarity. A general trend can enlighten the interaction of the adsorbed TPP: weak interaction on $\mathrm{Ag}$ and $\mathrm{Au}$; the intermolecular interactions result in attractive intermolecular forces, leading to the formation of close-packed islands with structures mainly following the molecular symmetry, while the adsorption on more strongly interacting substrates $(\mathrm{Cu}(110))$, though still related to attractive intermolecular forces, leads to more complex structures. In case of metal-free TPPs on strongly interacting substrates, intermolecular repulsion takes place. This trend is common to other porphyrins.

Confirmation of complex interaction frameworks at SAM interfaces is found in the weakly interacting porphyrinoid layers deposited on conductive substrates, such as $\mathrm{Au}(111)$ and graphite, HOPG [2], where dispersion is found to play a critical role in altering the adsorption and charge distribution. The comparative study between the two different substrates demonstrates that CoOEP bind more strongly to gold than to graphite, enlightening the molecule-substrate interactions in weakly bond interfaces and the role of dispersion forces between molecules and conductive substrates. Charge redistribution maps of both interfaces display charge localization mostly on the porphyrin molecule indicating a "push back effect" from the substrate, that is, a depletion of charge in the topmost layers. Density functional theory (DFT) calculations with dispersion indicate a larger substrate-to-molecule charge push on $\mathrm{Au}(111)$ than on HOPG (Figure 5). Moreover, comparison of density of states of isolated CoOEP molecule and its monolayer on gold-HOPG substrates revealed significant orbital hybridization and band shifts. Dispersion interactions played a critical role in altering the adsorption and charge distribution, especially in the $\mathrm{Au}(111)$ system. This framework could be valid also in other weakly bond organic-inorganic systems and should have important effects on the dynamical processes.
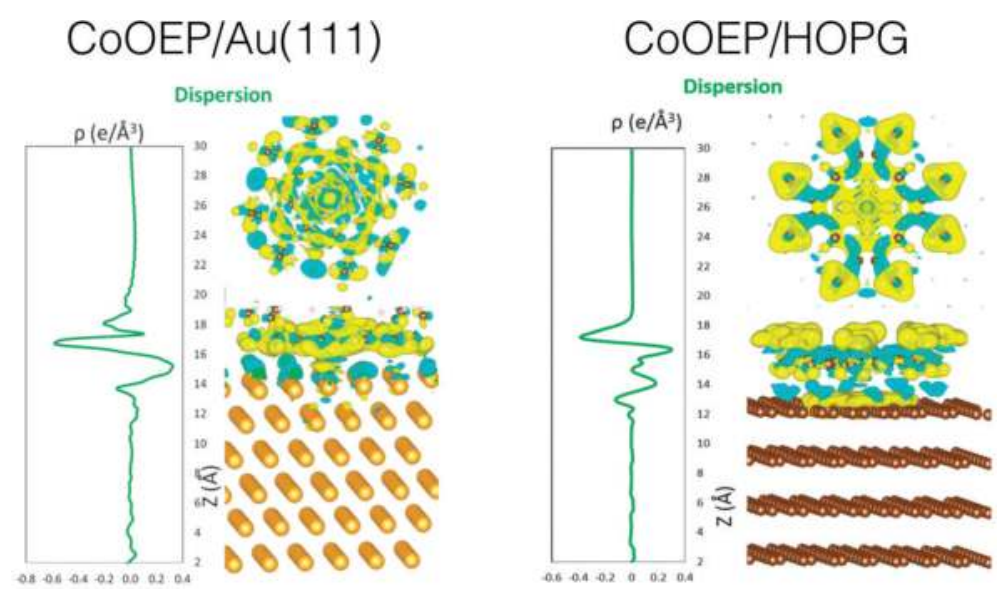

Figure 5. The charge density difference for CoOEP/Au(111) (left) and CoOEP/HOPG (right) systems. For each system, the panel on the right represents the 3D iso-density (+: yellow and -: cyan) of charge density difference. It is reproduced with permission from Ref. [2]. Copyright 2014, Royal Society of Chemistry. 
MPcs also show a trend. The tetrapyrrole SAM can self-organize into periodic ordered arrays on ordered surfaces, forming a variety of molecular 1D or 2D superstructures (see Tables 2 and 3 in Ref. [13]) and allowing for tunable anchoring sites. The MPc assembled in compact chains along the $\mathrm{Au}(110)$ reconstructed channels representing a dense single layer of molecules with 2D long range geometry where the molecules are all lying on the gold substrate and with little dispersion of the molecule-substrate distance. The interaction of MPc on the $\mathrm{Au}(110)$ surface is mediated by the $3 d$ metal states occupancy [39]: while $\mathrm{Ni}, \mathrm{Cu}$, and $\mathrm{Zn}$ metallic centers do not directly interact with the underlying Au substrate, transition-metal center atoms such as Fe and Co induce interaction states close to the Fermi level. The interaction of the regularly spaced Fe and Co metallic centers with a non-magnetic substrate, highly anisotropic $\mathrm{Au}(110)$, can break the symmetry of the metallic $d$ orbital carrying the magnetic moment, giving rise to reduced magnetic moments (Figure 6). In FePc and $\mathrm{CoPc}$, a charge transfer between the metal substrate and the molecular layer induces a redistribution of the molecular orbitals and structural reorganization of the anisotropic $\mathrm{Au}(110)$ substrate. In the case of $\mathrm{FePc} / \mathrm{Au}(110)$ system, it has been systematically shown how the interaction with the surface influences the ordering of the MPc states leading to novel interface properties. These results are intrinsically connected with the intermolecular and molecule-substrate interactions balance produced at interface.
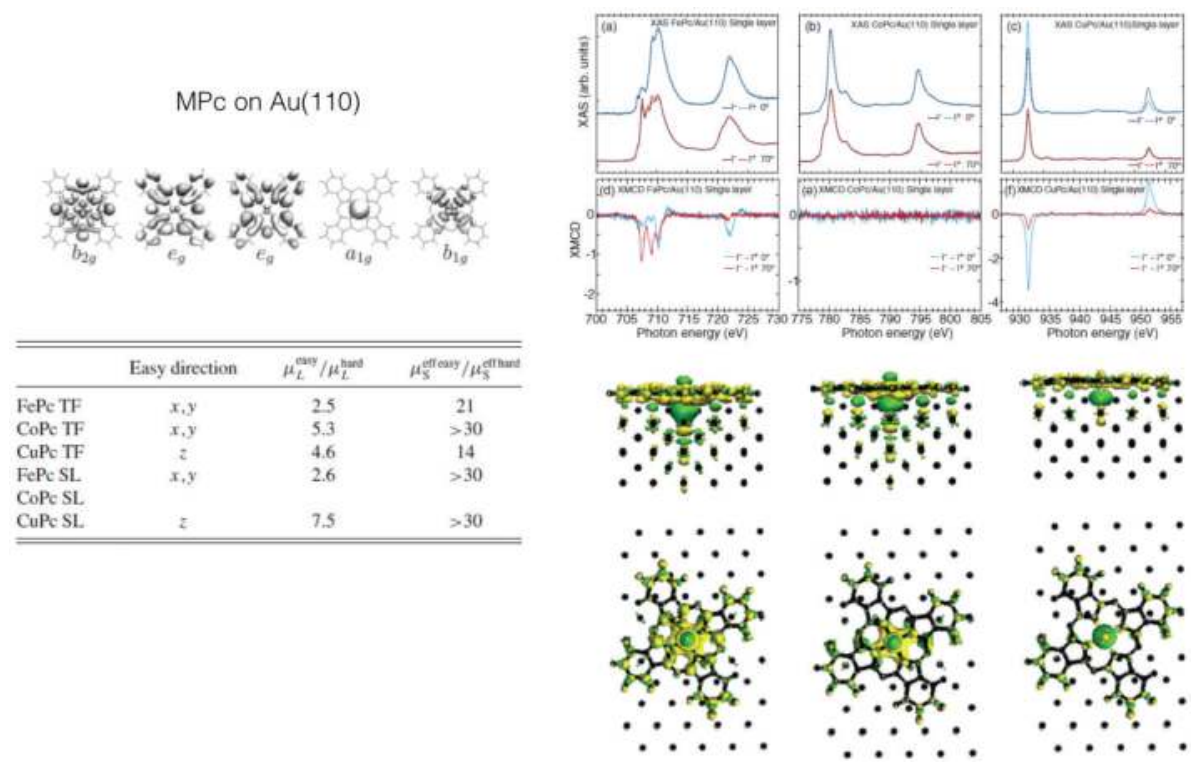

Figure 6. Circularly polarized X-ray absorption spectroscopy metal $\mathrm{L}_{2,3}$ spectra (a), (b), (c) and XMCD signals (d), (e), (f) for $\mathrm{FePc}, \mathrm{CoPc}$, and $\mathrm{CuPc}$ single layers, respectively, collected at 0 and $70^{\circ}$ of impinging photons and magnetic field (5 T) at $T=8 \mathrm{~K}$. The underneath panels report positive (green) and negative (yellow) charge density transfer upon adsorption of the three MPcs. The table reports the magnetic moments ratio between the easy and the hard magnetization axis at $B=5$ T, as measured by XMCD. Adapted with permission from Ref. [18], copyright 2012, American Physical Society. 


\section{Dynamics}

In the following paragraph, we will focus on a few selected examples to illustrate the power of the time-resolved investigation methods, when applied to the study of the electronic properties of gases or solid compounds.

For all the already-mentioned reasons, metallo-porphyrins and phthalocyanines are quite interesting systems. To engineer their electronic levels according to different applications either in photovoltaic devices or in opto-electronics (to mention some examples), it is fundamental to study in detail the mechanism of light absorption.

There are different de-excitation channels:

- a radiative decay, where the electron loses energy via the emission of photons of different wavelengths (fluorescence) and

- the creation of an "exciton", an electron-hole bound couple, whose energy is located inside the HOMO-LUMO band gap.

Excitons are crucial for transport in the organic compounds (Section 2.2). If the exciton binding energies are too high, about $1 \mathrm{eV}$, the bonding lengths are of the order of some Angstrom, so the exciton can move through the system before the recombination of positive and negative charges and thus get lost. But if the exciton is dissociated, that is the bond is broken, the two charges are free and can be collected at the electrodes of a photovoltaic device, for example.

So, several physical steps need to be characterized to engineer a proper molecule with the desired chemical and physical properties to be applied in an effective way to a working device, and time-resolved spectroscopies offer a new insight into all these processes.

Moreover, in order to apply molecular solids in new and fancy devices, based on a reversible chemical or structural modification induced by an external stimulus, like a flash of light, an electric field, or temperature or pressure variations, it is fundamental to understand how these mechanisms are activated. This family of compounds, named "dynamic molecular solids", is discussed in Ref. [16], where the author shows how fundamental is the control of electron, proton, and molecular motion. For example, a photo-induced molecular structural change could be used to control magnetic properties of the molecular solid.

\subsection{Dynamic time scales in solids}

What happens when a photon is absorbed? The first event after radiation absorption is an increase in energy of the system. In molecular aggregates after excitation, the molecule absorbing light has to redistribute the gained energy. It can be redistributed via different intramolecular processes, that is different channels that involve both atoms (nuclei vibrations) and electrons. All the possible processes have been deeply investigated at the 
beginning of 1900 by A. Jablonski. Depending on the amount of energy, the molecule can dissociate or isomerize (same atomic configuration but different geometry); otherwise, the energy is redistributed via different processes (see Figure 7 left):

- internal conversion (IC), which is the transition between electronic states of similar spin,

- intersystem crossing (ISC), which involves states of different spin multiplicities, and

- intramolecular vibration redistribution (IVR), that is vibrational energy flow from a given vibrational mode (or modes) to others.

All these processes are induced by modifications in the electronic structure, like the occupancy of the orbitals or the oxidation state. As can be seen also in Figure 7 (right), being related to electrons, all these phenomena take place on an ultrafast time scale.

In Figure 7 right are sketched several electronic/atomic processes and the time scales at which they manifest. Processes involving atoms' nuclei are typically on the pico/nano second time scale, while the time scale of electronic processes is in the femto/atto second region.

To access information on the electronic time scales, it is necessary to use investigation tools with comparable time duration [40] like ultrashort light pulses (of the order of a few tens of femtosecond) and proper wavelength, from the far infrared to the soft X-ray spectral region. With soft $X$-rays, it is possible to access the energy redistribution processes having the elemental selectivity for both the electronic structure and the geometric structure: the use of the ultrafast techniques to study MPcs and porphyrins has led, in the past decades, to an increased understanding of their photophysical and photochemical properties.

\subsection{Principles of selected time-resolved spectroscopies}

Since more than 100 years, researchers spent time in finding an investigation technique that was able to provide information on the time scale. In 1878, Leland Stanford, founder of the famous Stanford University, asked the photographer Eadweard Muybridge to demonstrate that while a horse is trotting or galloping, there is a moment in which the four legs are not touching the ground [41]. To prove this statement, the photographer recorded the first slow motion movie of history. He used 12 photocameras in raw that shot an image each, triggered
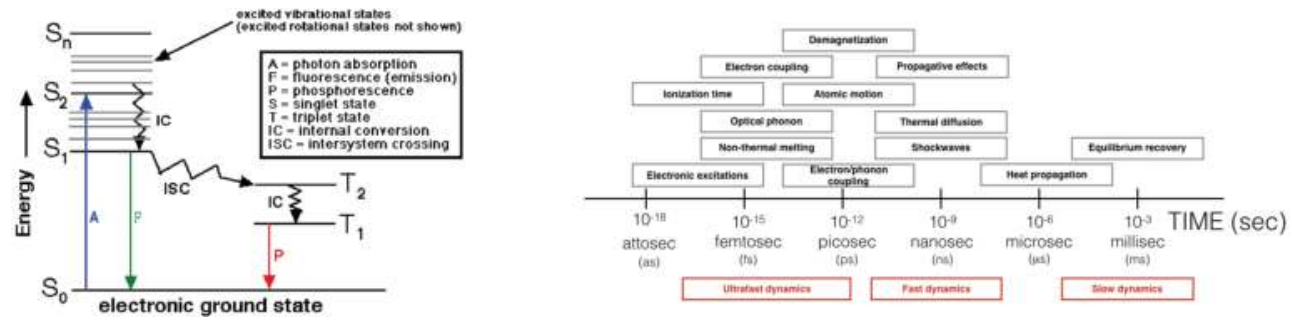

Figure 7. Jablonski diagram, from http://www.shsu.edu/chm_tgc/chemilumdir/JABLONSKI.html and dynamic processes time scales. 
by a wire that was pushed down by the wheel of the sulky, carrying the jockey, while it was passing in front of them.

From that time, big improvements have been made on the experimental setup to study the time evolution of a specific phenomenon. The limit of the high-speed photography reached few milliseconds $\left(10^{-3} \mathrm{~s}\right)$ in 1950 , but only with the advent of ultrashort laser light pulses at the end of 1990, it was possible to set up a new class of experiments with time resolution better than $10^{-9} \mathrm{~s}$ (nanoseconds). The limit of the $10^{-18} \mathrm{~s}$ (attoseconds) time scale was reached for the first time in 2001 by Krausz \& Co. [42]. With this kind of time resolution, it is now possible to observe and follow in real time chemical reactions on the electrons' time scales.

Going back to the starting example of the horse, we can say that a single picture of the horse is providing the structural characteristics of our sample or reaction or any other phenomenon, while the slow motion movie is providing its kinetics.

Several spectroscopic techniques are based on the following mechanism: a light pulse is used to excite the sample (pump), while a second light pulse is used to investigate what happened (probe). To realize the slow motion movie, we need to control the delay between the pump and the probe pulse, and this is done by varying the spatial distance the two beams have to travel. Since the probe has to reach the sample after the pump has excited it, the optical path of the probe has to be longer. Commonly, a computer-controlled delay line varies the path of the pump: when the two optical paths have exactly the same length and the two beams are perfectly overlapping, the light pulses are in temporal and spatial coincidence. This approach can be applied to several materials' characterization techniques. For practical reasons, it started with optical methods, since visible or infrared light pulses are quite easy to achieve with lasers. We are interested in soft X-ray-based techniques like photoelectron spectroscopy (PES). For the description of the technique, see Refs. [6, 37]. In the past 20 years, XUV light pulses became available in ordinary laboratories, thanks to ultrafast table top lasers, exploiting the high-order harmonic generation (HHG) process. For a description of the technique and of the experimental setup, see Refs. $[43,44]$ and references therein.

In a time-resolved photoemission (TR-PES) experiment, Figure 8, a pump pulse is used to excite electrons from filled to empty states, typically by means of an NIR/VIS or UV pulse. Subsequently, an XUV probe pulse is used to emit electrons from the ground state and from the excited state. The kinetic energy of the emitted electrons is then measured varying the delay between the pump and XUV probe pulses [45]. So far, such measurements have been mainly carried out with both the pump and probe energies from the near infrared to UV regimes (up to $\sim 6 \mathrm{eV}$ ). In this case, only electrons, which have interacted with photons from both pulses, will be emitted from the sample, and the technique is then often referred to as (time-resolved) two-photon photoemission spectroscopy (2PPE).

\subsection{Discussion on selected systems: gas phase dynamics in molecules, thin films, interfaces SAM}

The properties and thus the functions of the transition metal complexes (TMCs), even within a single molecular structure, depend specifically on the metal nested at their center. The 


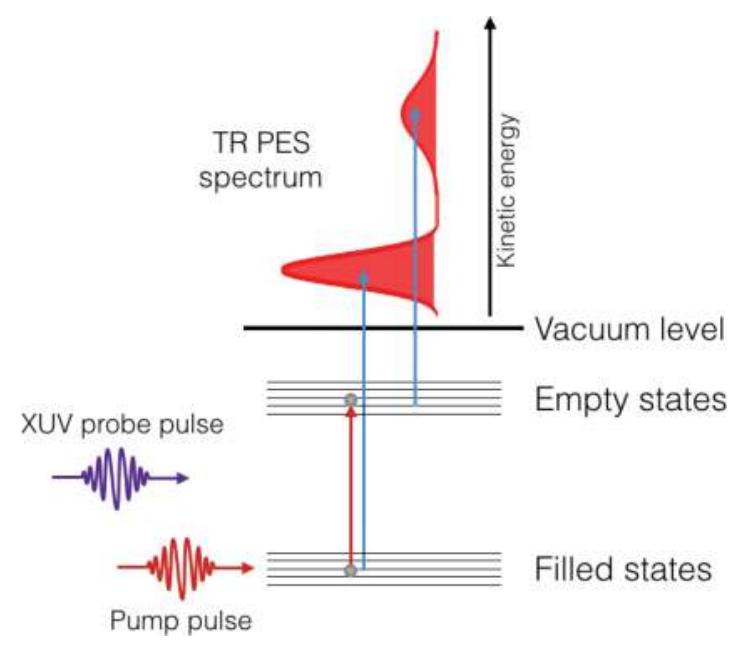

Figure 8. A sketch of the TR-PES process: the pump pulse is used to excite electrons from filled to empty states. Delayed in time, the XUV probe is used to emit electrons from the ground state and from the excited state. The kinetic energy of the emitted electrons is then measured. The measurement is then repeated several times varying the delay between the pump and XUV probe pulse, until the full dynamics of the process is mapped.

energetics and dynamics of the excited states differ among the various metal-porphyrins (characterized by different peripheral groups) and MPc and on the surrounding environment (the presence of a solvent or morphology). Quite critical is the dependence on the atomic number of the metallic atom and on whether the molecular complex is in solution or forms a thin film. Here some examples are discussed.

Gas phase: While the majority of the time-resolved measurements on metal complexes have been performed in solutions, only few literature works are related to the relaxation dynamics of molecules in gas phase. For tetrapyrrole molecules, early works have been published by Gouterman while recently the ultrafast dynamics of porphyrins in gas phase has been performed.

The relaxation dynamics of $\mathrm{CuTPP}, \mathrm{CuOEP}$, and $\mathrm{H}_{2} \mathrm{TPP}$ were investigated in pump-and-probe experiments [46] based on the optical absorption spectra (seen in Figure 2) of Cu porphyrins. The molecules were excited in the Soret B band using $3 \mathrm{eV}$ photon energy (400 nm), while the probe was a $4.5 \mathrm{eV}$ photon $(266 \mathrm{~nm})$. The energy diagram of the experiment is shown in Figure 8 bottom.

Upon excitation, the molecules relax showing different decay times, following the scheme of the picture: a first rapid decay $\left(t<100 \mathrm{fs}\right.$ ) from $\mathrm{S}_{2}$ to the charge transfer $(\mathrm{CT})$ state is followed by another rapid decay in the range of about $300 \mathrm{fs}-1$ ps to the triplet state, and a final long relaxation to the ground state takes place in the ns time scale. From the spectra of Figure 9 (top), it can be seen that the experimental data are fit with the convolution of three exponential curves to account for the three different decay times of the process. This behavior 

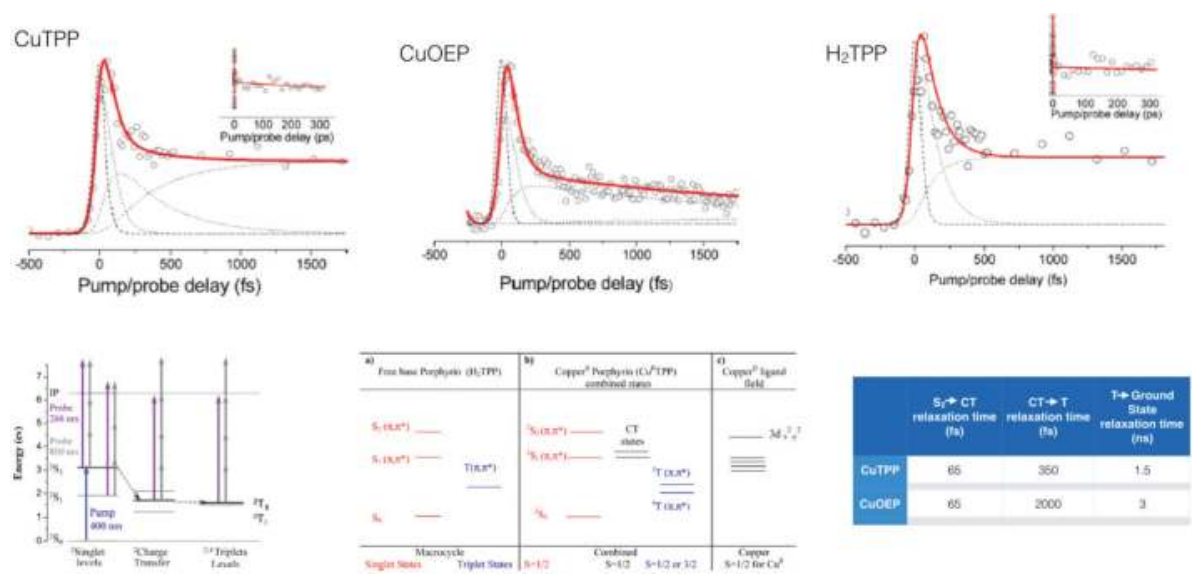

Figure 9. Top: CuTPP, CuOEP, and $\mathrm{H}_{2}$ TPP experimental pump-and-probe data. Bottom: (right) experiment energetics scheme, (middle) energy levels in CuTPP, CuOEP, and $\mathrm{H}_{2}$ TPP, and (left) table of excited states relaxation times. Adapted with permission from Ref. [49]. Copyright 2013, American Chemical Society.

remains similar to the porphyrin in solution demonstrating that the behavior of the molecular conformer in gas phase and in solution is similar.

As shown in the table of Figure 9, while the excitation dynamics in CuTPP and CuOEP presents the same decay time (65 fs) from the excited state $S_{2}$ to thecharge transfer (CT) state, the subsequent relaxations, first to the triplet states and finally to the ground state, are different: $350 \mathrm{fs}$ and $2000 \mathrm{fs}$ for CuTPP and CuOEP, and $1.5 \mathrm{~ns}$ and $3 \mathrm{~ns}$, respectively. This is due to the different decay pathways in the two molecules, that is to the different back-bond configuration induced by the peripheral groups in the two cases (see Section 2.1).

The excitation dynamics data of the $\mathrm{H}_{2} \mathrm{TPP}$ have to fit with the convolution of only two exponential curves because only two decay times, $S_{2} \rightarrow S_{1}$ and $S_{1} \rightarrow$ ground state, are present and their time constants are $110 \mathrm{fs}$ and $2 \mathrm{~ns}$, respectively. Clearly, in this case, the fast CT channel is missing because there is no metal atom in the middle.

Effects based on the different occupancies of the central metal $3 d$ states have been measured in porphyrins [47], while in the Zn complexes, the decay time of the $S_{2} \rightarrow$ CT shows long living excited states (600 fs); the analogous lifetimes in partially filled metal atoms result short of approximately one order of magnitude, up to about $50 \mathrm{fs}$ in FeOEP.

It is quite evident that even the presence of one extra electron in the central atom can lead to different behavior in the relaxation dynamics processes.

Thick films (intermolecular interactions perturb the dynamics): In this case, metallo-phthalocyanine $(\mathrm{CoPc})$ and metal-free Pc relaxation dynamics were investigated by means of pump-and-probe absorption spectroscopy in the UV-VIS spectral region [48]. The study revealed strong differences between the solution and thin films, with relevant dependence on the presence of the 
central metal atom. The samples have been resonantly optically excited (pump) in the $\mathrm{Q}$ band $(>600 \mathrm{~nm})$ and probed by a supercontinuum spectrum in the range of $(1200-460) \mathrm{nm}$, that is the whole absorption spectrum of the system was probed as a function of the delay time.

The effects induced by aggregation are visible in the optical absorption spectra. The measured decay times of Co complexes result much faster with respect to the metal-free phthalocyanine. The coupling between the $\pi$ orbitals and the $3 d$ orbitals provides an additional channel of energy relaxation (see Figure 10), as found in porphyrins and previously discussed. Moreover, in films, the intermolecular interactions take place together with the excitonic processes. These differences are enlightened in the optical absorption data, where a clear broadening of the $\mathrm{S}$ and $\mathrm{Q}$ bands is induced by the molecular aggregation. Actually, in case of CoPc, the singly occupied $3 d_{z^{\prime}}$ oriented orthogonal to the macrocycle plane, plays a major role. The related molecular orbital gives rise to delocalized exciton states that upon excitation result in resonance with the first excited state. This leads to an ultrafast charge transfer from the ligand excited state and the exciton. The absence of the $3 d$ orbitals in the $\mathrm{H}_{2}$ Pc inhibits this process and only two decay times are detectable.

Recently, in a study on the correlation between dynamics and exciton length in phthalocyanine films [49], valuable results are reported on the dependence of relaxation dynamics on the film's thickness in planar ZnPc and TiOPc (the latter presents an additional ligand orthogonal to the macrocycle plane). By means of TR-PES, it has been proved that the exciton transport is consistent with ballistic or coherent transport mechanisms in $\mathrm{ZnPc}$, while in TiOPc, the exciton transport mechanism is diffusive, due to the intramolecular hopping, absent in the first case.

As discussed previously, the molecular single layers deposited onto conductive substrates can lead to a plethora of bonds and molecule-substrate and intermolecular interactions that are specific of the systems. The dynamic study of the interface state's decay induced by the pump provides a detailed description of fundamental physical processes that govern the charge carrier dynamics at the interface.

As described in the previous section, TR-PES can provide information on the joint density of states evolution with time after the optical excitation, inducing for example HOMO-LUMO transition for selected pump energy. A nice example of TR-PES from molecular thin films of PCPDTBT deposited on ITO is presented in Ref. [48]. The measurements performed by
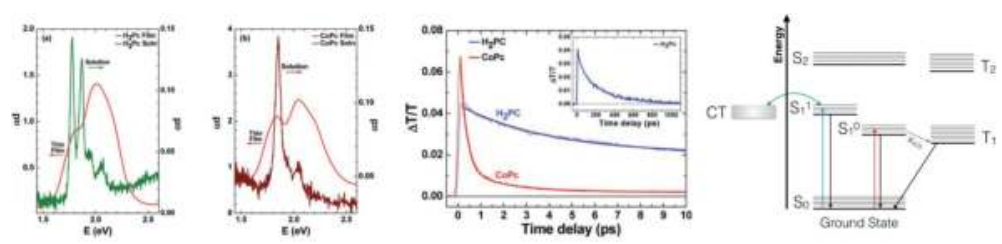

Figure 10. Left: Optical absorption spectra in film and solution for $\mathrm{H}_{2} \mathrm{Pc}$ and $\mathrm{CoPc}$, (middle) relaxation dynamics spectra for $\mathrm{H}_{2} \mathrm{Pc}$, and $\mathrm{CoPc}$ and (right) relaxation mechanisms for CoPc. Adapted with permission from Ref. [48]. Copyright 2010-2017, American Chemical Society. 
pumping at the two absorption resonance bands, $400 \mathrm{~nm}(3 \mathrm{eV})$ and $800 \mathrm{~nm}(1.5 \mathrm{eV})$, single out excitation of electrons from the HOMO to the LUMO orbitals as a function of the delay of the probe with respect to the pump, that is the filling and the subsequent de-excitation of the LUMO orbitals in the films.

Single Layers: The experimental study of single layer dynamics is quite challenging due to the very low signals involved. Only a few studies are present in the literature. The monolayer films onto coinage metal ordered surfaces can induce hybrid interface states in phthalocyanine systems. Harris and coworkers [50] compare the interfaces of $\mathrm{H}_{2} \mathrm{Pc}$ and FePc on $\mathrm{Ag}(111)$. The unoccupied interface state residing just above the Fermi level is the fulcrum of the investigation. From the experimental data, there is evidence on the formation of an interface state (IS in Figure 11) formed through a hybridization process of $\pi$ orbitals of the molecule and the surface state of the substrate. The formation of this hybrid interface state is slightly affected by the presence of the TM in the macrocycle that, as discussed in previous sections, chemically interacts with the substrate. Authors claim that the $\mathrm{FePc} / \mathrm{Ag}(111)$ results suggest that the interaction between the metal center and the $\operatorname{Ag}(111)$ surface is not strong enough to fundamentally alter the hybridization phenomenon.

This example demonstrates how TR-PES provides useful information on the unoccupied interface state and hence on the hybridization process.

The role of the substrate orientation in the photo-induced electron dynamics at the porphyrin/Ag interface [51] has been proved by pump-and-probe TR-PES experiments.

Due to the energy level alignment of the ZnTPP porphyrin on Ag(100), the first excited state is formed through the Ag-unoccupied sp bands. This configuration favors an indirect charge transfer path, from the substrate to the molecule during the pump-and-probe process, opening an excitation channel $250 \mathrm{fs}$ after the laser pump. The same timeresolved measurements carried out on porphyrin/Ag(111) show that in the latter case such an indirect path is not viable due to the different electronic configurations of the (111) interface.

In Figure 12 right are evidenced the two different electronic configurations, while on the left, the excitation process is sketched.
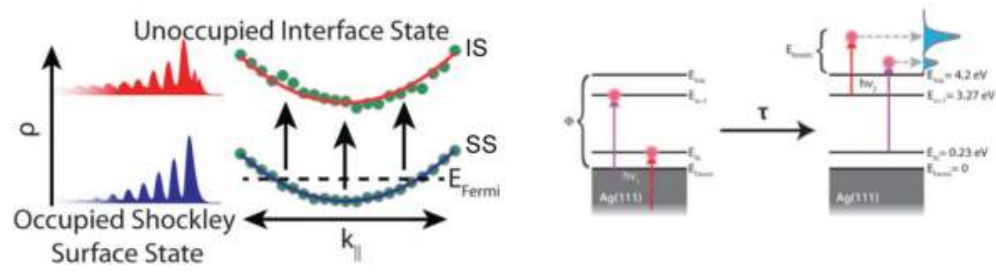

Figure 11. Left: Density of states for the occupied surface state and the unoccupied interface state and relative effective masses. Right: Schematic of the TR-PES process for $\mathrm{H}_{2} \mathrm{Pc} / \mathrm{Ag}(111)$ : An electron is pumped into an intermediate state by an infrared photon, and then, after a time delay, it is photoemitted by a UV photon and its kinetic energy is measured. Adapted with permission from Ref. [50]. Copyright 2014, American Chemical Society. 

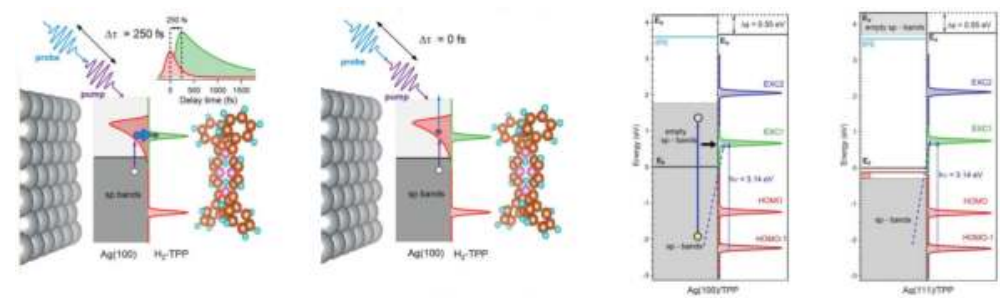

Figure 12. Left: An experimental set up of the pump-and-probe experiments. Right: A comparison of ZnTPP/Ag(100) and ZnTPP/Ag(111) electronic configurations. Adapted with permission from Ref. [51]. Copyright 2016, American Chemical Society.

The influence on the formation of the interface state, depending on the crystallographic orientation of the substrate, is also confirmed for other molecules, such as the PTCDA, by 2PPE experimental data [3], providing nice confirmation of the trend found by TR-PES.

Other important classes of experiments are related to TMC presenting spin cross-over processes in solution [52] and time-resolved X-ray absorption and diffraction [40]. Recently, time-resolved XPS core-level photoemission measurements performed on donor/acceptor blends of TMC organic molecules and fullerenes [53,54] have shown that the core levels experience binding energy shifts after the systems are excited by a femtosecond optical pump.

\section{Conclusions}

Control of the electronic levels is crucial for optoelectronic applications. Optical and magneto-electronic properties are intimately connected, and the accurate determination of electronic levels and de-excitation dynamics is mandatory in the optimization of electronic, photovoltaic, and spintronic devices. We have shown the interconnection between structural, electronic properties, and the relaxation dynamics in a single layer of transition metal complexes.

The study of ordered layers and SAMs on single crystal substrates allows for a detailed investigation of fundamental physical processes that govern the charge carrier dynamics at the interface. Although these systems differ from more disordered aggregates that resemble metal-organic contacts of real devices, their dynamical characterization completed by a robust determination of the electronic and structural properties can shed light on the processes governing important applications.

\section{Acknowledgements}

The Italian Eurofel program and the CITIUS project financed through Interreg Italia-Slovenija, 2007-2013, are acknowledged. Professor Giovanni De Ninno is also acknowledged for support and fruitful discussion. 


\section{Author details}

Maddalena Pedio ${ }^{1 *}$ and Barbara Ressel ${ }^{2}$

*Address all correspondence to: pedio@iom.cnr.it

1 CNR-IOM Laboratory TASC, Area Science Park, Basovizza, Trieste, Italy

2 University of Nova Gorica, Ajdovščina, Slovenia

\section{References}

[1] Geoffrey A. Lawrance. Introduction to coordination chemistry. John Wiley \& Sons, United Kingdom; 2009-2010. ISBN 9780470519301, Online ISBN 9780470687123, doi:10.1002/ 9780470687123

[2] Chilukuri B, Mazur U, Hipps KW. Effect of dispersion on surface interactions of cobalt(II) octaethylporphyrin monolayer on $\mathrm{Au}(111)$ and $\mathrm{HOPG}(0001)$ substrates: a comparative first principles study. Phys. Chem. Chem. Phys. 2014;16:14096. doi:10.1 039/c4cp01762e

[3] Marks M, Schöll A, Höfer U. Formation of metal-organic interface states studied with 2PPE. J. Electr. Spectrosc. Relat. Phenom. 2014;195:263-271. doi:10.1016/j.elspec. 2014.02.009

[4] Krause S, Casu MB, Schöll A, Umbach E. Determination of transport levels of organic semiconductors by UPS and IPS. New J. Phys. 2008;10:085001 (pp. 16). doi:10.1088/1367 $-2630 / 10 / 8 / 085001$

[5] Koch N, Ueno N, Wee Andrew TS, editors. The molecule-metal interface. Weinheim: Wiley-VCH Verlag GmbH \& Co KGaA; 2013. 255 p. doi:10.1002/9783527653171

[6] Zahn DRT, Gavrila GN, Salvan G. Electronic and vibrational spectroscopies applied to organic/inorganic interfaces. Chem. Rev. 2007;107:1161-1232. doi:10.1021/cr050141p

[7] DeLongchamp DM, Kline RJ, Fischer DA, Richter LJ, Toney MF. Molecular characterization of organic electronic films. Adv. Mater. 2010;XX:1-19.

[8] Gao Y. Surface analytical studies of interfaces in organic semiconductor devices. Mater. Sci. Eng. 2010;68:39-87. doi:10.1016/j.mser.2010.01.001

[9] Ishii H, Sugiyama K, Ito E, Seki K. Energy level alignment and interfacial electronic structures at organic/metal and organic/organic interfaces. Adv. Mater. 1999;11:605-625. doi:10.1002/(SICI)1521-4095(199906)11:8<605::AID-ADMA605>3.0.CO;2-Q

[10] Wang X-F, Tamiaki H. Cyclic tetrapyrrole based molecules for dye-sensitized solar cells. Energy Environ. Sci. 2010;3:94-106. doi:10.1039/b918464c 
[11] Green J, De Cleva P. Photoionization cross-sections: a guide to electronic structure. Coord. Chem. Rev. 2005;249:209-228. doi:10.1016/j.ccr.2004.02.012

[12] Bidermane I, Brumboiu IE, Totani R, Grazioli C, Shariati-Nilsson MN, Herper HC, Eriksson O, Sanyal B, Ressel B, de Simone M, Lozzi L, Brena B, Puglia C. Atomic contributions to the valence band photoelectron spectra of metal-free, iron and manganese phthalocyanines. J. Electr. Spectrosc. Rel. Phenom. 2015;205:92-97. doi:10.1016/j. elspec.2015.09.004

[13] Gottfried JM. Surface chemistry of porphyrins and phthalocyanines. Surf. Sci. Rep. 2015;70:259-379.

[14] Auwärter W, Écija D, Klappenberger F, Barth JV. Porphyrins at interfaces. Nat. Chem. 2015;7:105-120. doi:10.1038/NCHEM.2159

[15] Sirringhaus $\mathrm{H}$, et al. Two-dimensional charge transport in self-organized, high-mobility conjugated polymers. Nature. 1999;401:685-688. doi:10.1038/44359

[16] Sato Osamu. Dynamic molecular crystals with switchable physical properties. Nat. Chem. 2016;8:644-656. doi:10.1038/NCHEM.2547

[17] Senge MO, Ryan AA, Letchford KA, MacGowan SA, Mielke T. Chlorophylls, symmetry, chirality, and photosynthesis. Symmetry. 2014;6:781-843. doi:10.3390/sym6030781

[18] Gargiani P, Rossi G, Biagi R, Corradini V, Pedio M, Fortuna S, Calzolari A, Fabris S, Cezar JC, Brookes NB, Betti MG. Spin and orbital configuration of metal phthalocyanine chains assembled on the $\mathrm{Au}(110)$ surface. Phys. Rev. B. 2013;87:165407. doi:10.1103/ PhysRevB.87.165407

[19] Bartolomé J, et al. Chapter 9: Magnetism of metal phthalocyanines. In J. Bartolomé, et al. editors, Molecular magnets. Heidelberg: Springer; 2014. ISBN 9783642406096

[20] Denys Nautschenko, M. Pedio unpublished

[21] Krasnikov SA, Preobrajenski AB, Sergeeva NN, Brzhezinskaya MM, Nesterov MA, Cafolla AA, Senge MO, Vinogradov AS. Electronic structure of $\mathrm{Ni}$ (II) porphyrins and phthalocyanine studied by soft X-ray absorption spectroscopy. Chem. Phys. 2007;332:318-324. doi:10.1016/j.chemphys.2006.12.015

[22] Miedema PS, de Groot FMF. The iron L edges: Fe 2p X-ray absorption and electron energy loss spectroscopy. J. Electr. Spectr. Rel. Phenom. 2013;187:32-48. doi:10.1016/j. elspec.2013.03.005

[23] Jurow M, Schuckman AE, Batteas JD, Drain CM. Porphyrins as molecular electronic components of functional devices. Coord. Chem. Rev. 2010;254:2297-2310. doi:10.1016/j. ccr.2010.05.014

[24] Rossi G, d'Acapito F, Amidani L, Boscherini F, Pedio M. Local environment of metal ions in phthalocyanines: K-edge X-ray absorption spectra. Phys. Chem. Chem. Phys. 2016;18: 23686-23694. doi:10.1039/c6cp04022e 
[25] Baerends EJ, Ricciardi G, Rosa A, van Gisbergen SJA. A DFT/TDDFT interpretation of the ground and excited states of porphyrin and porphyrazine complexes. Coord. Chem. Rev. 2002;230:5-27. doi:10.1016/S0010-8545(02)00093-0

[26] Kroll T, Kraus R, Schönfelder R, Aristov V Yu, Molodtsova O V, Hoffmann P, Knupfer $\mathrm{M}$. Transition metal phthalocyanines: Insight into the electronic structure from soft x-ray spectroscopy J. Chem. Phys. 2012;137:054306. doi: 10.1063/1.4738754

[27] Umari P, Fabris S. Importance of semicore states in GW calculations for simulating accurately the photoemission spectra of metal phthalocyanine molecules. J. Chem. Phys. 2012;136:174310 (6). doi:10.1063/1.4705360

[28] Nobuo Ueno "Electronic Structure of Molecular Solids: Bridge to the Electrical Conduction" 2012; pp. 65-89 in Physics of Organic Semiconductors, Second Edition. Wiley-VCH Verlag GmbH \& Co KGaA; doi: 10.1002/9783527654949.ch3

[29] Djuric T, Ules TH, Gusenleitner S, Kayunkid N, Plank H, Hlawacek G, Teichert C, Brinkmann M, Ramsey M, Resel R. Substrate selected polymorphism of epitaxially aligned tetraphenyl-porphyrin thin films. Phys. Chem. Chem. Phys. 2012;14:262-272. doi:10.1039/c1cp22299f

[30] Marsili M, Umari P, Di Santo G, Caputo M, Panighel M, Goldoni A, Kumar M, Pedio M., Solid state effects on the electronic structure of $\mathrm{H}_{2} \mathrm{OEP}$, Phys. Chem. Chem. Phys. 2014;16:27104-27111.

[31] Stoll LK, Zgierski MZ, Kozlowski PM. Density functional theory analysis of nickel octaethylporphyrin ruffling. J. Phys. Chem. A. 2002;106:170-175.

[32] Ryuzaki S, Hasegawa T, Onoe J. , Effects of inter-molecular charge-transfer excitons on the external quantum efficiency of zinc-porphyrin/C60 heterojunction photovoltaic cells, J. Appl. Phys. 2009;105:113529.

[33] Schreiber F. Structure and growth of self-assembling monolayers. Progr. Surf. Sci. 2000;65:151-256.

[34] Resta A, Felici R, Kumar M, Pedio M. Ni and Cu octaethyl porphyrins ordered monolayer on $\mathrm{Au}(111)$ surfaces. J. Non-Cryst. Solids. 2010;356:1951-1954. doi:10.1016/j. jnoncrysol.2010.05.051

[35] Hipps KW. Scanning Tunneling Spectroscopy. In Handbook of applied solid state spectroscopy, D.R. Vij, editor. New York: Springer-Verlag; 2006. ISBN 0-387-32497-6

[36] Pedio M, Cepek C, Felici R. Organic molecules on noble metal surfaces: the role of the interface. Dr. Yen-Hsun Su, editor. Noble metals. InTech; 2012. doi: 10.5772/34551. Retrieved from http://www.intechopen.com/books/noble-metals/organic-molecules-onnoble-metal-surfaces-the-role-of-the-interface

[37] Koch N, Gerlach A, Duhm S, Glowatzki H, Heimel G, Vollmer A, Sakamoto Y, Suzuki $\mathrm{T}$, Zegenhagen J, Rabe JP, Schreiber F. Adsorption-induced intramolecular dipole: correlating molecular conformation and interface electronic structure. J. Am. Chem. Soc. 2008;130:7300-7304. doi:10.1021/ja800286k 
[38] Lodi Rizzini A, Krull C, Mugarza A, Balashov T, Nistor C, Raoul Piquerel R, Klyatskaya S, Ruben M, Sheverdyaeva PM, Moras P, Carbone C, Stamm CH, Miedem PS, Thakur PK, Sessi V, Soares M, Yakhou-Harris F, Cezar JC, Stepanow S, Gambardella P. Coupling of single, double, and triple-decker metal-phthalocyanine complexes to ferromagnetic and antiferromagnetic substrates. Surf. Sci. 2014;630:361-374. doi:10.1016/j.susc.2014.07.008

[39] Gargiani P, Angelucci M, Mariani C, Betti MG. Metal-phthalocyanine chains on the $\mathrm{Au}(110)$ surface: interaction states versus $d$-metal states occupancy. Phys. Rev. B. 2010;81:085412. doi: 10.1103/PhysRevB.81.085412

[40] see for example Cammarata M, Bertoni R, Lorenc M, Cailleau H, Di Matteo S, Mauriac C, Matar SF, Lemke H, Chollet M, Ravy S, Laulhé C, Létard J-F, Collet E. Sequential activation of molecular breathing and bending during spin-crossover photoswitching revealed by femtosecond optical and x-ray absorption spectroscopy. Phys. Rev. Lett. 2014;113:227402. doi: and refs therein

[41] https://alumni.stanford.edu/get/page/magazine/article/?article_id=39117

[42] Hentsche M, Kienberger R, Spielmann CH, Reider GA, Milosevic N, Brabec T, Corkum P, Heinzmann U, Drescher M, Krausz F. Attosecond metrology. Nature. 2001;414:509_ 513. doi: $10.1038 / 35107000$

[43] Pfeifer T, Spielmann C, Gerber G. Femtosecond x-ray science. Rep. Prog. Phys. 2006; 69:443-505. doi:10.1088/0034-4885/69/2/R04

[44] Grazioli C, Callegari C, Ciavardini A, Coreno M, Frassetto F, Gauthier D, Golob D, Ivanov R, Kivimäki A, Mahieu B, Bucar B, Merhar M, Miotti P, Poletto L, Polo E, Ressel B, Spezzani C, De Ninno G. CITIUS: an infrared-extreme ultraviolet light source for fundamental and applied ultrafast science. Rev. Sci. Instr. 2014;85:023104. doi:10.1063/1.4864298

[45] Cappel UB, Plogmaker S, Terschlüsen JA, Leitner T, Johansson EMJ, Edvinsson T, Sandell A, Karis O, Siegbahn H, Svensson S, Mårtensson N, Rensmo H, Söderström J. Electronic structure dynamics in a low bandgap polymer studied by time-resolved photoelectron spectroscopy. Phys. Chem. Chem. Phys. 2016:18;21921-21929. 2016. doi:10.1039/ C6CP04136A

[46] Ha-Thi MH, Shafizadeh N, Poisson L, Soep B. An efficient indirect mechanism for the ultrafast intersystem crossing in copper porphyrins. J. Phys. Chem. A., 2013;117: 8111-8118. doi:10.1021/jp4008015

[47] Shafizadeh N, Ha-Thi M-H, Poisson L, Soep B. Ultrafast electronic relaxation of excited state of biomimetic metalloporphyrins in the gas phase. In Dr. Lilyana Pramatarova, editor. On biomimetics. InTech; 2011. doi:10.5772/19976. Retrieved from http://www. intechopen.com/books/on-biomimetics/ultrafast-electronic-relaxation-of-excited-stateof-biomimetic-metalloporphyrins-in-the-gas-phase

[48] Gadalla A, Crégut O, Gallart M, Hönerlage B, Beaufrand J-B, Bowen M, Boukari S, Beaurepaire E, Gilliot P. Ultrafast optical dynamics of metal-free and cobalt phthalocyanine thin films. J. Phys. Chem. C. 2010;114: 4086-4092. doi:10.1021/jp911438y 
[49] Wang T, Kafle TR, Kattel B, Chan W-L. Observation of an ultrafast exciton hopping channel in organic semiconducting crystals. J. Phys. Chem. C. 2016;120:7491-7499. doi:10.1021/ acs.jpcc.6b01400

[50] Caplins BW, Suich DE, Shearer AJ, Harris JB. Metal/phthalocyanine hybrid interface states on Ag(111). J. Phys. Chem. Lett. 2014;5:1679-1684. doi:10.1021/jz500571z

[51] Tognolini S, Ponzoni S, Sedona F, Sambi M, Pagliara S. Role of the substrate orientation in the photoinduced electron dynamics at the porphyrin/Ag interface. J. Phys. Chem. Lett. 2015;6:3632-3638. doi:10.1021/acs.jpclett.5b01528

[52] Chergui M. On the interplay between charge, spin and structural dynamics in transition metal complexes. Dalton Trans. 2012;41:13022. doi:10.1039/c2dt30764b

[53] Arion T, Neppl S, Roth F, Shavorskiy A, Bluhm H, Hissain Z, Gessner O, Eberhardt W., Site-specific probing of charge transfer dynamics in organic photovoltaics Appl. Phys. Lett. 2015;106:121602. doi: 10.1063/1.4916278

[54] Ozawa K, Yamamoto S, Yukawa R, Akikubo K, Emori M, Sakama H and Matsuda I, Capturing transiently charged states at the $\mathrm{C}_{60} / \mathrm{TiO}_{2}(110)$ interface by time-resolved soft X-ray photoelectron spectroscopy Org. Electron., 2016;31:98-103. doi: 10.1016/j.orgel.2016. 01.020 